\title{
Efectos del trote progresivo en la sesión de educación física sobre el porcentaje de grasa corporal y VO2máx en estudiantes con sobrepeso y obesidad. Prueba Piloto
}

Effects of progressive jogging in the Physical Education session on the percentage of body fat and $\mathrm{VO}_{2}$ max in students with overweight and obesity. Pilot test

Lenin Tlamatini Barajas Pineda

Facultad de Ciencias de la Educación. Universidad de

Colima, México

lenin_barajas@ucol.mx

(DD https://orcid.org/0000-0003-0975-8144

Pedro Julián Flores Moreno

Facultad de Ciencias de la Educación. Universidad de

Colima, México

pedrojulian_flores@ucol.mx

(iD) https://orcid.org/0000-0003-2587-513X

Adriana Isabel Andrade Sánchez

Facultad de Ciencias de la Educación. Universidad de

Colima, México

isa_andrade@ucol.mx

(D) https://orcid.org/0000-0001-9790-5719

Javier Arturo Hall López

Facultad de Deportes. Universidad Autónoma de Baja

California, México

javierhall@uabc.edu.mx

(iD https://orcid.org/0000-0002-7808-0181

José E. Del Río Valdivia

Facultad de Medicina. Universidad de Colima, México

delriojose@ucol.mx

Uriel Andrés Viera Maldonado

Facultad de Ciencias de la Educación. Universidad de

Colima, México

jperez57@ucol.mx

Jenny Daniela Pérez Mojica

Facultad de Ciencias de la Educación. Universidad de

Colima, México

uviera@ucol.mx 


\section{Resumen:}

El presente estudio cuasi experimental y prospectivo tubo como objetivo determinar si el trote progresivo a 10 minutos durante la sesión de educación física caracterizada por desarrollar actividades moderadas a vigorosas, tenía efecto sobre el porcentaje de grasa corporal y el $\mathrm{VO}_{2}$ máx en estudiantes con sobrepeso y obesidad. La población fue de 59 niños (57.63\%) y niñas (42.37\%) organizados en grupo control y experimental. Entre los principales resultados de esta investigación posterior a cuatro meses de intervención, fueron que las niñas y los niños del grupo experimental disminuyeron el porcentaje de grasa corporal de manera significativa ( $\mathrm{p} .<0.005)$ y ambos grupos (control y experimental), aumentaron significativamente la capacidad aeróbica máxima (p. $<0.005)$.

Palabras Clave: Educación Física, Trote progresivo, Sobrepeso y Obesidad.

\section{ABstract:}

The present quasi-experimental and prospective study aimed to determine if 10-minute progressive jogging during the Physical Education session characterized by developing moderate to vigorous activities had an effect on the percentage of body fat and VO2max in overweight and obese students. The population under study was 59 boys $(57.63 \%)$ and girls (42.37\%) organized in control and experimental groups. Among the main results of this research after four months of intervention, we found that girls and boys in the experimental group significantly decreased the percentage of body fat (p. $<005)$ and that both groups (control and experimental) significantly increased maximum aerobic capacity (p. <005).

KEYWORDS: Physical Education, Progressive trot, Overweight and obesity.

\section{INTRODUCCIÓN}

Según la Organización Mundial de la Salud (OMS, 2021) la obesidad y el sobrepeso se definen como la acumulación anormal o excesiva de grasa que puede ser perjudicial para la salud, poseer dicho desbalance energético es un factor de riesgo para padecer numerosas enfermedades crónicas, entre las que se incluyen las cardiovasculares, la diabetes mellitus tipo II, trastornos del aparato locomotor, algunos tipos de cáncer, entre otras (Dávila-Torres, González-Izquierdo y Barrera-Cruz, 2015). Además, existe una estrecha relación entre la obesidad abdominal, la resistencia a la insulina para el desencadenamiento de anormalidades metabólicas que dan paso al síndrome metabólico (Lizarzaburu, 2013).

Además de los beneficios que genera la actividad física, se ha identificado que pacientes que cuentan con obesidad, hipertensión, diabetes mellitus tipo II, y bajo nivel de actividad física presentan un mayor riesgo de contagio de enfermedades respiratorias agudas. Por lo que las recomendaciones actuales con respecto a la actividad física se enfocan en realizar actividad física moderada - vigorosa de forma repetitiva con volúmenes de tiempo de 30 a 60 minutos con lo que es posible mantener un peso corporal adecuado y mejorar la actividad de vigilancia de patógenos reduciendo así la morbilidad y mortalidad por enfermedades respiratorias agudas (Hamer, et al., 2020; Nieman, 2020, Denay, et al., 2020). A lo anterior descrito, se suma lo explicado por Lopez - Gatell, (2020), que la combinación de enfermedades metabólicas representa un factor de riesgo de mortalidad alto al contraer COVID 19. Por lo tanto un estilo de vida sedentario, limitado por actividad física e inadecuada alimentación, genera el aumento del cumulo de tejido adiposo y por lo tanto la obesidad, la cual se identifica como el problema de salud pública mundial más graves del siglo vigésimo primero, reconocida como la gran pandemia que afecta indistintamente a mujeres y a hombres, adultos, adolescentes y niños (Mercado y Vilchis, 2013; Dávila-Torres, González-Izquierdo y Barrera-Cruz, 2015), no exclusivo de países económicamente desarrollados pues está afectando progresivamente a países de bajos y de medianos ingresos, sobre todo en el medio urbano (OMS, 2020),

$\mathrm{Al}$ respecto, México ocupa el primer lugar con la tasa de mayor obesidad infantil en el mundo y el segundo entre adultos (UNICEF, 2019), ya que evidencias estadísticas relacionadas a la población infantojuvenil, indican una prevalencia de sobrepeso y obesidad en escolares en ascenso desde 1999 (Shamah-Levy et al., 2018).Los datos mas actuales en población escolar de 6 a 11 años, indican una prevalencia del $34.4 \%$ y en la población adolescente de 12 a 19 años del 35\%, considerándose una de las más altas del mundo.

Además de la alta prevalencia de obesidad en la población de edad escolar, la inactividad física es denominada como la insuficiente participación en la actividad física durante el tiempo de ocio y a un aumento de los comportamientos sedentarios durante las actividades laborales y domésticas, así como también, el uso 
de los medios de transporte pasivos (OMS, 2015). Evidencia científica indica que la falta de actividad física es una de las causas para la aparición de sobrepeso, obesidad y síndrome metabólico (Blanchard et al., 2013; Dávila-Torres, González-Izquierdo y Barrera-Cruz, 2015). En México, sólo el 17.2\% de la población de 10 a 14 años cumplen con la recomendación de actividad física de la OMS - de 60 minutos diarios de actividades físicas de intensidades moderadas a vigorosas en mayor proporción de tipo aeróbica por lo menos tres veces a la semana -, además, la prevalencia de inactividad física moderada a vigorosa en adolescentes de 15 a 19 años es de casi del 40\% (Shamah-Levy et al., 2016). La actividad física en un contexto escolar, se contempla en la asignatura de educación física, la cual se entiende como una forma de intervención pedagógica que contribuye a la formación integral de las niñas, los niños y adolescentes al desarrollo de su motricidad e integrar su corporeidad (SEP, 2017, p.161). En los últimos 30 años de presencia en la educación básica en México, se ha promovido el desarrollo de hábitos de higiene, sana alimentación, cuidado del cuerpo, mejoramiento de las capacidades física, por lo tanto, se ha considerado a la escuela como el espacio propicio para crear hábitos de vida saludable (SEP, 2009, p.181). Sin embargo, investigaciones en los últimos años indican que las actividades dentro de las sesiones de educación física no logran los niveles de intensidad moderada a vigorosa recomendada y el compromiso motor de los escolares ha sido pobre e insuficiente contabilizandose un promedio de $20 \mathrm{~min}$ de tiempo compromiso motor y hasta $22 \mathrm{~min}$ de actividad moderada vigorosa dentro de la clase de educación física la cual tiene un tiempo destinado de 50 min dos veces por semana dentro del jornada escolar. (Salazar, et al. 2017; Flores, et al., 2017; Hall-López, et al., 2018).

La promoción de actividad física moderada o vigorosa y la disminución de estilos de vida sedentarios junto con una dieta saludable y adecuada, son acciones indispensables para prevenir y controlar el sobrepeso y la obesidad en la infancia y la adolescencia (Dávila-Torres, González-Izquierdo, y Barrera-Cruz, 2015), dichas promociones acompañadas de un de proceso pedagógico son una estrategia importante para esa población en particular (Northrup, Cottrell, y Wittberg, 2008).

Por lo anterior el propósito de esta investigación fue el determinar el efecto del trote progresivo de 10 minutos en la sesión de educación física sobre el porcentaje de grasa corporal y la capacidad aeróbica máxima en niñas y niños de una escuela primaria de la comunidad rural Suchitlán, del municipio de Comala, México.

\section{Metodología}

\section{Participantes}

La investigación se llevó acabo a finales del año 2019 y principios del 2020 (interrumpido por la contingencia de la pandemia de la COVID 19), bajo un diseño metodológico cuasi experimental (Bernal, 2010), por su planeación corresponde a un estudio prospectivo (Manterola et al., 2019) y longitudinal (Arnau y Bono 2008), con muestreo no probabilístico por conveniencia (Thomas, 2001), solicitando la anuencia de participación a los directivos y profesores de los seis grados de 4to, 5to y 6to, grupos "A" (Control) y B" (Experimental), siguiendo los principios éticos de investigación en seres humanos de la declaración de Helsinki (Puri, et al., 2009).

De 127 niñas y niños de entre 9 y 11 años de edad, fueron seleccionados 31 para el grupo Control ( $58.1 \%$ hombres, $41.9 \%$ mujeres) y 28 para el Grupo Experimental ( $57.1 \%$ hombres, $42.9 \%$ mujeres), quienes cumplieron con los criterios de inclusión: sobrepeso u obesidad en atención a los resultados del IMC, permiso y consentimiento informado firmado por padres de familia, sin padecimientos cardiovasculares. Aunque todos los niños siguieron participando y recibiendo las sesiones de educación física fueron excluidos de los análisis estadísticos y resultados de esta investigación quienes no cumplieron con los criterios anteriormente citados. 
Los sujetos del grupo control desarrollaron las sesiones de la sesión de educación física con actividades moderadas a vigorosas más un trote progresivo de 10 minutos durante 4 meses, es decir, comenzaron a trotar a partir de 30 segundos y fueron aumentando el tiempo de trote conforme el avance de las sesiones de educación física hasta llegar a un total 10 minutos como tiempo máximo, manteniéndolo así hasta finalizar la intervención.

\section{Instrumentos}

Los parámetros antropométricos fueron valorados previo a la intervención y posterior a ella, respetando los protocolos y normas de la Asociación Internacional de Kinantropometria (ISAK) por sus siglas en inglés. Para valorar el peso $(\mathrm{kg})$ se utilizó la bascula digital Tanita Um-081, y la estatura $(\mathrm{cm})$, fue determinada con el estadimetro portátil Seca 213. Utilizando la información anterior, se determinó el Índice de Masa Corporal (IMC) como indicador del estado nutricional a través de la fórmula de Quetelet: $(\mathrm{IMC}=\operatorname{peso}(\mathrm{kg}) /$ estatura $(\mathrm{cm})$.

El porcentaje de grasa corporal fue determinada a partir de la toma de los pliegues cutáneos del tríceps y pierna medial con el plicómetro Slim Guide, siguiendo el protocolo de la ISAK y una vez obtenidos los resultados se utilizó la ecuación de Slaughter et al. (1988, citado en Hall et al., 2017), que permite determinar el porcentaje de grasa corporal de niñas y niños con un error de estimación del 3.8\%. Clasificando los resultados en: Muy bajo, Bajo, Rango Óptimo, Moderadamente Alto, Alto y Muy Alto.

- Niños \% masa grasa $=0.735 \times$ (pliegue tricipital + pliegue pierna medial $)+1,0$

- Niñas \% masa grasa $=0.610 x$ (pliegue tricipital + pliegue pierna medial $)+5,1$

Para determinar la capacidad aeróbica máxima se utilizó la prueba de campo de Course Navette de 20 metros, en la que las niñas y los niños deberían de desplazarse sobre un espacio de 20 metros en ida y vuelta esperando para arrancar el estímulo en forma de sonido; la velocidad alcanzada y la edad se introducen en la ecuación de Léguer et al. (1984, citado en Hall et al., 2017) que proporciona de manera indirecta el consumo máximo de oxígeno.

- $\mathrm{VO}_{2}$ máx $=31.025+3.238 \mathrm{X}-3.248 \mathrm{~A}+0.1536 \mathrm{AX}$, siendo $\mathrm{X}$ la velocidad a la que se paró el sujeto y $\mathrm{A}$ la edad.

La intensidad del trote de los 10 minutos se controló mediante el Test Talk, que es un método subjetivo de evaluación de la intensidad mediante la capacidad de hablar del individuo durante una actividad física determinada. El Test Talk posee correlaciones altas con mediciones objetivas y subjetivas, como la frecuencia cardiaca, consumo máximo de oxígeno etcétera, lo cual es un test confiable en su uso, incluso con pacientes que poseen alguna alteración cardiovascular (Quinn y Coons, 2011).

- Consiste en tres etapas: la primera es la etapa positiva (+), donde los sujetos pueden hablar confortable y cómodamente, tiene un promedio de $52 \%$ de FCRu y su intensidad de actividad física es baja.

- La segunda etapa o etapa intermedia (+-) es donde los sujetos tienen una intensidad moderada, un promedio de $79 \%$, es esta etapa los sujetos no están seguros de hablar cómodamente.

- Por último, está la tercer etapa o etapa negativa (-), donde alcanzan una FCRu de 83\%, la intensidad es alta y no pueden hablar cómodamente o totalmente (Quidequeo et al., 2015).

Se decidió empezar el trote desde 30 segundos progresivo hasta llegar a los 10 minutos, debido a que las niñas y los niños al principio, se les complicaba correr, ya que la mayoría lo hacia de forma explosiva, por lo que se cansaban inmediatamente después de iniciar el trote. Durante cada clase se fue aumentando 30 segundos 
al trote y mientras se realizaba la actividad se explicaba como se debía de correr con relación a la intensidad, mecánica del movimiento, cadencia y respiración, así mismo se utilizaron componentes motivacionales.

La intensidad de la actividad física de las sesiones de educación física se evaluó mediante el Sistema para Observar el Tiempo de Instrucción de Actividad Física por sus siglas en inglés (SOFIT) (McKenzie, Sallis \& Nader, 1992); se evaluaron todas las sesiones de educación física de los grupos control y experimental, eligiendo al azar a cuatro estudiantes de cada sesión ( 2 hombres y 2 mujeres) que se observaron en secuencia rotatoria de 12 intervalos durante 20 segundos cada uno, repitiéndose las observaciones durante toda la sesión, siguiendo un audio que indicó los tiempos de registro de actividad, utilizando para ello un reproductor MP3 Samsung. Para determinar la intensidad de la sesión de educación física se usaron categorías para clasificar los niveles de actividad, permitiendo estimar el gasto energético asociada a la actividad física, este procedimiento se clasificó en cinco categorías: 1) acostado, 2) sentado, 3) parado, 4) caminando, y 5) muy activo que corresponde a correr o una actividad con gasto energético mayor. Estos códigos han sido calibrados monitoreando los latidos del corazón y el sistema ha sido validado en sesiones de educación física usando acelerómetros Caltrac (Sharma et al., 2011). A partir de la cuantificación de estos códigos se estableció el índice de actividad física moderada a vigorosa (IAFMV) sumando porcentualmente los códigos 4) caminando y 5) muy activos, del total del tiempo de la sesión de educación física.

\section{Análisis estadístico}

El análisis estadístico se realizó mediante el Paquete Estadístico para las Ciencias Sociales (SPSS), versión 22.0. Para realizar el análisis de la normalidad de los datos y así determinar los estadísticos que permitieran realizar las comparativas entre los datos y cumplir los objetivos de la investigación, se utilizó la prueba Kolmogorov-Smirnov con resultados significativos $(\mathrm{p} \leq 0.05)$, motivo por el cuál se utilizó el test W de Wilcoxon para comparar los resultados intragrupos (de la pre y post intervención tanto del grupo control como en el experimental) y el test estadístico U de Mann Whitney para comparar los resultados entre grupos (control vs experimental).

\section{Resultados}

Se trabajó con las niñas y los niños de la escuela primaria Gorgonio Ávalos T.V. de la comunidad rural de Suchitlán, Comala, Colima, de los últimos grados escolares. De los 59 alumnos, 15 cursan cuarto grado $(6$ del grupo A y 9 del grupo B), 16 quinto grado (7 del grupo A y 9 del grupo B) y 28 de sexto grado (15 del grupo A y 13 del grupo B). En total el $57.63 \%$ eran hombres y el $42.37 \%$ mujeres (Ver Tabla 1 ). Es importante aclarar que los grupos son organizados por la dirección del plantel al momento que el alumno entra a la primaria, y como investigadores y profesores no se tiene la inferencia para la modificación de los mismos. 
TABLA 1

Análisis descriptivo del sexo y edad de los grupos control y experimental

\begin{tabular}{|l|c|c|c|}
\hline \multicolumn{4}{|c|}{ Tabla 1 } \\
Análisis descriptivo del sexo y edad de los grupos control y experimental \\
\hline Variable & Grupo Control & Gripo Experimental & Total \\
& N & N & N \\
& 31 & 28 & 59 \\
\hline Sexo & $\mathrm{M}=58.1 \%$ & $\mathrm{M}=57.1 \%$ & $\mathrm{M}=57.63 \%$ \\
& $\mathrm{~F}=41.9 \%$ & $\mathrm{~F}=42.9 \%$ & $\mathrm{~F}=42.37 \%$ \\
\hline Edad & 10.19 años $( \pm 0.87)$. & 10.25 años $( \pm 0.79)$. & 10.22 años $( \pm 0.83)$. \\
\hline \multicolumn{4}{|c|}{ Elaboración propia. } \\
\hline
\end{tabular}

Fuente: Elaboración propia.

El peso en kilogramos del grupo control aumentó de manera significativa entre la pre y post intervención ( $\mathrm{p}$-valor 0.000), así como también ocurrió con el grupo experimental ( $\mathrm{p}$ - valor 0.005), sin embargo, no hubo diferencias estadísticas significativas entre la pre y post intervención con relación al peso corporal en kilogramos entre grupo control y experimental (Ver Tabla 2).

TABLA 2

Promedio de Peso de grupo control y experimental

\begin{tabular}{|c|c|c|c|c|c|c|c|c|c|}
\hline \multicolumn{4}{|c|}{ Control } & \multicolumn{4}{|c|}{ Experimental } & \multicolumn{2}{|c|}{$p$-valor } \\
\hline \multirow[t]{2}{*}{$\mathbf{n}$} & Pre & Post & $p^{a}$ & $\mathbf{n}$ & Pre & Post & $p^{2}$ & & \\
\hline & $k g( \pm$ d.e. $)$ & $k g( \pm$ d.e. $)$ & & & $k g( \pm$ d.e. $)$ & $k g( \pm$ d.e. $)$ & & Pre & Post \\
\hline 31 & $44.5( \pm 9.09)$ & $46.1( \pm 9.36)$ & 0.000 & 28 & $46.8( \pm 11.07)$ & $47.9( \pm 10.63)$ & 0.005 & 0.514 & 0.762 \\
\hline \multicolumn{10}{|c|}{$\begin{array}{l}\text { p-valor resultante de la prueba W de Wilcox on comparando el peso en la primera toma (pre) y en la } \\
\text { segunda toma (post). } \\
\text { p-valor resultante de la prueba U de Mann Whitney comparando el peso el grupo control (B) y el } \\
\text { experimental (A). }\end{array}$} \\
\hline
\end{tabular}

Fuente: Elaboración propia.

El porcentaje de grasa corporal en el grupo control en pre y post intervención no tuvo diferencia estadística significativa ( $\mathrm{p}$-valor 0.650 ) mientras que en el grupo experimental en pre y post intervención si presentó diferencia estadística significativa (p-valor 0.030), (Ver Tabla 3). 
TABLA 3

Promedio del porcentaje de grasa corporal de grupo control y experimental.

\begin{tabular}{|c|c|c|c|c|c|c|c|c|c|}
\hline \multicolumn{4}{|c|}{ Control } & \multicolumn{3}{|c|}{ Experimental } & & \multicolumn{2}{|c|}{$p-v a l o r^{b}$} \\
\hline \multirow[t]{2}{*}{$\mathbf{n}$} & Pre & Post & $p^{2}$ & $\mathbf{n}$ & Pre & Post & $p^{2}$ & & \\
\hline & $\%( \pm$ de $)$ & $\%( \pm$ d.e. $)$ & & & $\%( \pm$ d.e. $)$ & $\%( \pm$ de $e)$ & & Pre & Post \\
\hline 31 & $25.3( \pm 6.49)$ & $25.04( \pm 6.81)$ & 0.650 & 28 & $26.3( \pm 8.08)$ & $25.07( \pm 7.63)$ & 0.030 & 0.756 & 0.849 \\
\hline
\end{tabular}

a. p-valor resultante de la prueba $\mathrm{W}$ de Wilc oxon comparando el peso en la primera toma (pre) y en la segunda toma (post).

b. p-valor resultante de la prueba U de Mann Whitney comparando el peso el grupo control (B) y el experimental (A).

Fuente: Elaboración propia

Fuente: Elaboración propia

La capacidad aeróbica máxima entre el pre y post intervención del grupo control fue estadísticamente significativa ( $\mathrm{p}$-valor 0.009 ) así como también resultó para el grupo experimental ( $\mathrm{p}$-valor 0.001), sin embargo, no hubo diferencias entre ambos grupos en pre y post intervención (Ver Tabla 4).

TABLA 4

Promedio de $\mathrm{VO}_{2}$ máx de grupo control y experimental

\begin{tabular}{|c|c|c|c|c|c|c|c|c|c|}
\hline \multicolumn{4}{|c|}{ Control } & \multicolumn{4}{|c|}{ Experimental } & \multicolumn{2}{|c|}{$p$-valor $r^{b}$} \\
\hline \multirow[t]{2}{*}{$\mathbf{N}$} & Pre & Post & $p^{2}$ & $\mathbf{n}$ & Pre & Post & $p^{2}$ & & \\
\hline & $\%( \pm$ d.e. $)$ & $\%( \pm$ d.e. $)$ & & & $\%( \pm$ dee. $)$ & $\%( \pm$ dee $)$ & & Pre & Post \\
\hline 31 & $39.2( \pm 3.88)$ & $41.1( \pm 2.85)$ & 0.009 & 28 & $38.8( \pm 4.20)$ & $41.7( \pm 3.72)$ & 0.001 & 0.674 & 0.717 \\
\hline
\end{tabular}

a. p-valor resultante de la prueba $\mathrm{W}$ de Wilcox on comparando el peso en la primera toma (pre) y en la segunda toma (post).

b. p-valor resultante de la prueba U de Mann Whitney comparando el peso el grupo control (B) y el experimental (A).

Fuente: Elaboración propia

En cuanto a la intensidad de las sesiones de educación física, para el grupo control y experimental del 4to grado fue de 54.54\%; para el grupo control y experimental del 5 to grado la intensidad de las sesiones de educación física fue de $51.08 \%$ y para los grupos control y experimentales de Gto grado fue de 50.06\%; por lo que se cumple con al menos mas de la mitad de la sesión de actividades moderadas a vigorosas. Cabe señalar que las sesiones de educación física fueron planeadas de acuerdo a los aprendizajes esperados del Programa de Estudios Aprendizaje Clave 2017, y tanto los grupos experimentales como los controles recibieron la misma sesión respectivamente por grado escolar, (Ver Tabla 5). 
TABLA 5

Porcentaje del tiempo de compromiso motor en las sesiones de educación física

\begin{tabular}{|c|c|c|c|c|}
\hline $\begin{array}{c}\text { Grados y } \\
\text { Grupos }\end{array}$ & $\begin{array}{c}\text { Cuarto } \\
\text { Grado “Ay } \\
\text { B" }\end{array}$ & $\begin{array}{c}\text { Quinto } \\
\text { Grado “Ay } \\
\text { B" }\end{array}$ & $\begin{array}{l}\text { Sexto Grado } \\
\text { "Ay B" }\end{array}$ & Total \\
\hline Promedio & $\begin{array}{c}\%( \pm \text { d.e. }) \\
54.54( \pm 7.25)\end{array}$ & $\begin{array}{c}\%( \pm \text { d.e. }) \\
51.08( \pm 12.82)\end{array}$ & $\begin{array}{c}\%( \pm \text { d.e. }) \\
50.06( \pm 8.15)\end{array}$ & $\begin{array}{c}\%( \pm \text { d.e. }) \\
51.899( \pm 9.87)\end{array}$ \\
\hline
\end{tabular}

Fuente: Elaboración propia

\section{Discusión}

El objetivo de este estudio fue determinar si el trote progresivo a 10 minutos en la sesión de educación física, la cual estaba caracterizada por desarrollar actividades moderadas a vigorosas, tenía efectos sobre porcentaje de grasa corporal y la capacidad aeróbica máxima en niñas y niños con sobrepeso y obesidad. Entre los principales resultados de esta investigación posterior a cuatro meses de intervención, fueron que las niñas y los niños del grupo experimental disminuyeron el porcentaje de grasa corporal de manera significativa y ambos grupos (control y experimental), aumentaron significativamente la capacidad aeróbica máxima.

Con relación al promedio de peso corporal, ambos grupos aumentaron de peso, experimental $(1.1 \mathrm{~kg}) \mathrm{y}$ control (1.6kg), este hecho puede deberse al crecimiento natural de las y los niños, pues en atención a los percentiles de crecimiento de peso con relación a la edad de la OMS (2007ab), se observa que en el transcurso de tres meses pueden llegar aumentar hasta un kilogramo de peso en promedio. Sin embargo, es importante resaltar que el aumento de peso para el grupo experimental no es debido a la acumulación de grasa corporal, pues en su caso, disminuyeron su porcentaje, de $26.3 \mathrm{~kg}( \pm 8.08)$ a $25.07 \mathrm{~kg}( \pm 7.63)$, ( $\mathrm{p}$-valor $=0.03)$.

La grasa corporal es dependiente del género, edad y origen étnico de los niños y adolescentes (Daniels et al., 1997), en este sentido, la población en la que se intervino fue mixta (hombres y mujeres), de origen mexicano, con edades de entre 9 y 11 años, todos ellos, clasificados con sobrepeso y obesidad en atención a su edad y género por su al IMC. El porcentaje de grasa corporal promedio general comparado del grupo control y experimental en pre intervención sumó $51.6 \%$ ( $\mathrm{p}$-valor $=0.756$ ) y posterior a la intervención fue de $50.11 \%$ ( $\mathrm{p}$-valor $=0.849$ ), es decir, una diminución no significativa general del $1.49 \%$, sin embargo, el promedio del porcentaje de grasa del grupo experimental en pre y post intervención sí disminuyó de manera significativa ( $\mathrm{p}$-valor $=0.030)$, situación que no ocurrió en el grupo control ( $\mathrm{p}$-valor $=0.650)$, estos resultados fueron similares a los reportados por Hall et al. (2017) con relación a una reducción significativa del porcentaje de grasa corporal promedio de $28.0 \% \pm 5.1(\mathrm{p}$-valor $=0.022)$ posterior a un programa de actividad física moderada a vigorosa de diez meses bajo un modelo denominado CATCH.

Acorde con lo anterior, se puede manifestar que el efecto de trote progresivo a 10 minutos más la sesión de educación física caracterizada por desarrollar actividades moderadas a vigorosas coadyuvan a la disminución de los porcentajes de grasa corporal del de las niñas y los niños de una escuela primaria de una comunidad rural, y en este sentido, ambos protocolos se proponen como una alternativa para lo que solicita el estudio de Dávila-Torres, González-Izquierdo y Barrera-Cruz (2015), con relación a implementar acciones de atención inmediata para la prevención y control de la obesidad en niños, adolescentes y adultos, particularmente en sexo femenino de zonas rurales pues en su caso, son aún más vulnerables por el aumento de sobrepeso y obesidad en menor tiempo que los hombres (Shamah-Levy et al., 2018). Con relación a la capacidad aeróbica 
máxima, tanto el grupo control $(41.1 \pm 2.85$, p-valor $=0.009)$, así como el grupo experimental $(41.7 \pm 3.72$, p-valor $=0.001)$ aumentaron de manera significativa, en este sentido, las sesiones de educación física con actividades moderadas a vigorosas desarrolladas durante cuatro meses jugaron un papel importante; los datos anteriores, son similares a los reportado en el estudio de Hall et al. (2017), desarrollado en el mismo contexto, reportando un aumento significativo de $\mathrm{VO}_{2}$ máx promedio de $36.3 \pm 2.8 \mathrm{ml} / \mathrm{kg}^{-1} / \mathrm{min}^{-1}$ ( -valor $=0.001)$ a través de actividades físicas moderadas a vigorosas bajo un modelo denominado CATCH. En otra investigación desarrollada por Welk et al. (2011), se demostraron mejoras significativas en la capacidad aeróbica en niños con sobrepeso y obesidad quienes participaron en ejercicio físico, educación física, actividad física o iniciación deportiva durante seis meses. Existen otras intervenciones en las que se han observado valores altos de capacidad aeróbica máxima con la presencia en menor medida de obesidad, enfermedades metabólicas y cardiovasculares (Ruiz et al., 2011 en Hall et al., 2017).

$\mathrm{Al}$ comparar los resultados sobre el índice de actividad física moderada a vigorosa en las sesiones de educación física de nuestra investigación, la cuál fue $51.89 \%$ ( \pm 9.87$)$ con otras investigaciones realizadas en México, se reportan valores distintos; Pérez (2009), indica no haber logrado ni el 50\% del total de las sesiones con dicho índice, por su parte, Jennings-Aburto et al. (2009), lograron sólo un índice de 29.2\%, Flores, et al. (2017) reporta un $18.10 \%$, mientras que en el ámbito rural el índice fue $19 \%$ según lo reportado por Salazar et al. (2017), el reporte que más se acerca es el de Hall et al. (2018), en el que se logró un índice de $41 \%$. En este sentido, es fundamental que el docente organice y planee sesiones de educación física en la que el compromiso motor, definido por Sierra (2003) como la parte del tiempo disponible para la práctica las cuales deberán de ser con intensidades de moderadas a vigorosas la mayor parte del tiempo de la sesión, como lo sugiere la United States National Association for Sport and Physical Education (NASPE), (Banville, 2006 en Hall-López et al., 2018), cabe destacar que las actividades diseñadas en esta investigación además de ser consecuentes con los aprendizajes esperados del programa de estudios vigente, si logró obtener resultados positivos sobre los niveles de actividad física. No obstante, y con relación a los datos mencionados con anterioridad, la gran mayoría de las intervenciones relacionadas con el compromiso motor y niveles de actividad física recomendada por organismos internacionales en la sesión de educación física son muy pocas (Kobel et al., 2015), por lo que este espacio se convierte en un área de oportunidad para los educadores físicos, los cuales no deben de consumir el tiempo de la sesión en actividades de gestión, organización o demostración (Travieso y Pavón, 2006).

En cuanto a la Actividad Física, sólo el $17.2 \%$ de la población de 10 a 14 años cumplen con la recomendación de actividad física de la OMS, además, la prevalencia de inactividad física moderada a vigorosa en adolescentes de 15 a 19 años es de casi el 40\% de la población.

\section{Conclusiones.}

En conclusión, el trote progresivo a 10 minutos y sesiones de educación física con actividades moderadas a vigorosas desarrolladas en 4 meses mejoran de manera significativa la capacidad aeróbica y reduce el porcentaje de grasa corporal en niñas y niños. Sin embargo, es fundamental para futuras investigaciones, aumentar el periodo de intervención, implementar una evaluación intermedia y acompañar el proyecto con asesoría nutricional.

\section{Agradecimientos}

Al Mtro. Carlos Ceballos, director del plantel, a los docentes de grupo Paula, Erick, Adriana, Jaime, Julieta y Bertha, a los padres de familia, niños y niñas de la primaria, así como a Javier por apoyar el proceso y aportar al crecimiento profesional de Uriel y Yenny. 


\section{REFERENCIAS}

Arnau, J. y Bono, R. (2008). Estudios longitudinales. Modelos de diseño y análisis. Escritos de Psicología, 2(1), 32-41.

Banville, D. (2006). Analysis of exchanges between novice and cooperating teachers during internships using the NCATE/NASPE standards for teacher preparation in physical education as guidelines. Research Quarterly for Exercise and Sport, 77(2), 208-221.

Bernal, C. (2010). Metodología de la investigación. México: Editorial Mc

Blanchard, C., Shilton, T. y Bull, F. (2013). Global Advocacy for Physical Activity (GAPA): global leadership towards a raised profile. Global Health Promotion, 77(2), 208-221.

Daniels, S., Khoury, P. y Morrison, J. (1997). The utility of body mass index as a measure of body fatness in children and adolescents: differences by race and gender. Pediatrics, 99(6), 804-807

Dávila-Torres, J., González-Izquierdo, J. y Barrera-Cruz, A. (2015). Panorama de la obesidad en México. Rev Med Inst Mex Seguro Soc., 52(2), 240-9

Denay, K. L., Breslow, R. G., Turner, M. N., Nieman, D. C., Roberts, W. O. y Best, T. M. (2020). ACSM call to action statement: COVID-19 considerations for sports and physical activity. Current sports medicine reports, 19(8), 326-328.

Flores, M., Margarita, S., Gómez, F., Barreto, V., Valdovinos, G., Vicente, R. y Del Río, E. (2017). Medición del tiempo efectivo de la clase de educación física y su impacto en el gasto calórico en escolares de nivel primaria del municipio de Colima, México. Sportis, 3(1), 34-49. doi: https://www.doi.org/10.17979/sportis.2017.3.1.17

Hall-López, J., Ochoa-Martínez, P., Macías, C., Zuñiga, B. y Sáenz-Lopez, B. (2018). Actividad física moderada a vigorosa en educación física y recreo en estudiantes de primaria y secundaria de la frontera México-USA. Sportis, 4(3), 426-442.

Hall-López, J., Ochoa-Martínez, P., Zamudio, B., Sánchez, L., Uriate, G., Almagro, T., Moncada-Jiménez, J. y SáenzLópez, B. (2017). Efecto de un programa de actividad física moderada a vigoroza de diez meses sobre el VO2Máx y el porcentaje de grasa corporal en niños con sobrepeso y obesidad. MHSalud, 14(1), 1-12. doi: https://www .doi.org/10.15359/mhs.14-1.6

Hamer, M., Kivimäki, M., Gale, C. R., \& Batty, G. D. (2020). Lifestyle Risk Factors for Cardiovascular Disease in Relation to COVID-19 Hospitalization: A Community-Based Cohort Study of 387,109 Adults in UK. medRxiv, 1. 1-11. doi: https://www.doi.org/10.1101/2020.05.09.20096438

Jennings-Aburto, N., Nava, F., Bonvecchio, A., Safdie, M., Gonzalez-Casanova, I., Gust, T.y Rivera, J. (2009). Physical activity during the school day in public primary schools in Mexico City. Salud Publica Mex., 51(2), 141-147.

Lizarzaburu R, J. (2013). Síndrome metabólico: concepto y aplicación práctica. An Fac med, 74(4), 315-320.

Manterola, C., Quiroz, G., Salazar, P. y García, N. (2019). Metodología de los tipos y diseños de estudio más frecuentemente utilizados en investigación clínica. Revista Médica Clínica Las Condes, 30(1), 36-49.

Mckenzie, T. L., Sallis, J. F. y Nader, P. R. (1992). Sofit - System for Observing Fitness Instruction Time. Journal of Teaching in Physical Education, 11(2), 195-205.

Mercado, P. y Vilchis, G. (2013). La obesidad infantil en México. Alternativas en psicología, 17(28), 49-57.

Nieman, D. C. (2020). Coronavirus Disease-2019: a tocsin to our aging, unfit, corpulent, and immunodeficient society.J Sport Health Sci, 9(4), 293-301. doi: https://www.doi.org/10.1016/j.jshs.2020.05.001

Northrup, K. L., Cottrell, L. A., y Wittberg, R. A. (2008). L.I.F.E.: a school-based heart-health screening and intervention program. J Sch Nurs, 24(1), 28-35. doi: https://www.doi.org/10.1622/1059-8405(2008)024[002 8:LASHSA]2.0.CO;2

Oganización Mundial de la Salud (2021). Obesidad y sobrepeso. Recuperado de https://www.who.int/es/news-room /fact-sheets/detail/obesity-and-overweight

Organización Mundial de la Salud. (2015). Inactividad física: un problema de salud pública mundial. Boletin Epidemiologico. 
Organización Mundial de la Salud. (2017a). Peso para la edad en niños. Recuperado de https://www.who.int/growth ref/cht_wfa_boys_perc_5_10years.pdf?ua=1

Organización Mundial de la Salud. (2017b). Peso para la edad en niñas. Recuperado de https://www.who.int/growt href/cht_wfa_girls_perc_5_10years.pdf?ua $=1$

Pérez, B. A. (2009). Impacto de la clase de educación física sobre la actividad moderada y vigorosa en niños de primaria. Revista Mexicana de Investigación en Cultura Física y Deporte, 1(1), 150-172.

Puri, K. S., Suresh, K. R., Gogtay, N. J. y Thatte, U. M. (2009). Declaration of Helsinki, 2008: implications for stakeholders in research. Journal of Postgraduate Medicine, 55(2). doi: https://www.doi.org/10.4103/0022-38 59.52846

Quidequeo, D., Ibarra, J., San Martin, M. y Monroy, M. (2015). Validez del Talk Test como método para estimar la intensidad de ejercicio en un grupo de uniformados sanos entre 18 y 25 años de la comuna de Valdivia. Revista de Estudiosos en Movimiento, 2(1), 41-46.

Ruiz, J.R., Castro-Pinero, J., Espana-Romero, V., Artero, E. G., Ortega, F. B., Cuenca, M. M. y Castillo, M. J. (2011). Field-based fitness assessement in youg people: the ALPHA Health-related fitness test battery for children and adolescents. British Journal of Sports Medicine, 45(6), 518-524.

Salazar, C., Moreno, F., Valencia, M., Valencia, Ch. y Camacho, A. (2017). Evaluación del tiempo efectivo de la clase de educación física en escolares del ámbito rural. Revista Edu-fisica.com., 9(19), 34-46.

SEP. (2017). Aprendizajes Clave. Para la educación integral. Aprendizajes Clave. Plan y programas de estudio para la educación básica.

Shamah-Levy, T., Cuevas-Nasu, L., Gaona-Pineda, E., Gómez-Acosta, L., Morales-Rúan, M., Hernández-Ávila, M. y Rivera-Dommarco, J. (2018). Sobrepeso y obesidad en niños y adolescentes en México, actualización de la Encuesta Nacional de Salud y Nutrición de Medio Camino 2016. Salud Pública de México, 60(3), 244-253. doi: https://www.doi.org/10.21149/8815

Shamah-Levy, T., Cuevas-Nasu, L., Rivera-Dommarco, J. y Hernández-Ávila, M. (2016). Encuesta Nacional de Salud y Nutrición de Medio Camino 2016. (ENSANUT MC 2016). Instituto Nacional de Salud Pública y Secretaría de Salud, 2016(Ensanut), Recuperado de https://www.gob.mx/cms/uploads/attachment/file/209093/ENSA NUT.pdf\%0Ahttps://www.gob.mx/cms/uploads/attachment/file/209093/ENSANUT

Sharma, S., Chuang, R. J., Skala, K., y Atteberry, H. (2011). Measuring physical activity in preschoolers: Reliability and validity of The System for Observing Fitness Instruction Time for Preschoolers (SOFIT-P). Meas Phys Educ Exerc Sci, 15(4), 257- 273. doi: https://www.doi.org/10.1080/1091367X.2011.594361

Sierra, A. (2003). Actividad fisica y salud. El compromiso Fisiológico en la clase de eduación física. Sevilla: Wanceulen.

Thomas, J.R., Nelson, J.K., Silverman, S. y Silverman, S.J. (2001). Research Methods in Physical Activity (6 Ed.). Champaign, Ilinois: Human Kinetics.

Travieso, G. y Pavón, R. (2006). Valoración de la utilidad del tiempo en las clases de educación física de tercer graado en municipio Las Tunas. Efdeportes.com Recuperado de https://www.efdeportes.com/efd100/tiempo.htm

Unicef. (2019). Estado Mundial de la Infancia 2019. Informe niños, alimentos y nutrición: crecer bien en un mundo cambiante. Recuperado de https://www.unicef.org/mexico/informes/estado-mundial-de-lainfancia-2019\#: :text=En\%20M\%C3\%A9xico\%20el\%205\%25\%20de,aumentando\%20en\%20todo\%20el $\% 20$ mundo.

Welk, G. J., De Saint-Maurice Maduro, P.F., Laurson, K. R. y Broyn, D. D. (2011). Field evaluation of the new FITNESSGRAM $^{\circ}$ criterion-referenced standards. American Journal of Preventive Medicine, 41(2), 131-142. 